\title{
A Cost-Based Model for Data Repairing/in Cloud Database
}

\author{
V. Naresh, G. Balaji, Aseen Babu, S.Lakshmi
}

\begin{abstract}
Trustworthiness vital based information fixing is an iterative method comprising of two sections: become aware of and bunch errors that damage given uprightness requirements (ICs); and alternate esteems internal every accumulating with the quit purpose that the altered database fulfills the ones ICs. Be that as it is able to, maximum existing programmed arrangements deal with the manner towards identifying and gathering blunders direct (e.G., infringement of utilitarian situations using string uniformity), whilst putting greater consideration on heuristics of converting traits interior each gathering. In this paper, we advise a reexamined semantics of infringement and data consistency w.R.T. A variety of ICs. The amended semantics depends on string likenesses, rather than commonplace techniques that usage syntactic mistake identity using string equity. Alongside the changed semantics, we likewise advise another price model to degree the expense of facts restore by thinking about separations between strings. We display that the modified semantics gives a large trade to better distinguishing and collecting mistakes, which accordingly improves both exactness and overview of the accompanying records solving step. We reveal that discovering least value fixes within the new version is NP-difficult, in any event, for a solitary FD. We devise gifted calculations to find out surmised fixes. Likewise, we create documents and streamlining techniques to improve the proficiency. Examinations show that our methodology altogether beats existing programmed restoration calculations in both accuracy and assessment.
\end{abstract}

Keywords:Cloud computing, service-level agreements, Database integration, Matching dependencies, functional dependencies, integrity constraints

\section{INTRODUCTION}

Allotted computing offers IT administrations to customers via the net. Disbursed computing empowers the facilitating of unavoidable applications. It depends on a pay-greater simplest as prices rise up model; in this way, it offers to marketers. For example, it takes into account the slow improvement of a task from little scale to huge scale belongings.

One of the contrasts between disbursed computing foundation and unique registering frameworks is the affiliation of virtualization innovation. Therefore, the cloud has a virtualization layer, on the same time because the opportunity processing frameworks don't have this sort of layer [1]. Conventional software program provisioning

Revised Manuscript Received on 14 September, 2019.

V. Naresh, Assist. Professor, Department of CSE, Siddhartha Institute of Technology \& Sciences, Narapally, Ghatkesar, Hyderabad, Telangana, India.

G. Balaji, Assist. Professor, Department of CSE, Siddhartha Institute of Technology \& Sciences, Narapally, Ghatkesar, Hyderabad, Telangana, India.

Aseen Babu, Assist. Professor, Department of CSE, Siddhartha Institute of Technology \& Sciences, Narapally, Ghatkesar, Hyderabad, Telangana, India.

S.Lakshmi, Assistant Professor, Department of CSE, Kingston Engineering College, Vellore, Tamilnadu, India. models dole out man or woman application components to figuring hubs. A extremely good outline of a cloud is various that has a solitary preparing center. There may be a need of simultaneously launching VMs on that host. Notwithstanding the truth that practically speaking VMs are setting confined, the VMs want to proportion the getting prepared centers. Consequently, the diploma of gadget belongings reachable to each VM is forced via the whole gadget belongings handy within the host. CloudSim take a look at tool [2] bolsters VM provisioning at two tiers: have degree and VM degree. At have level, it's far doable to decide the amount of the all out managing depth of every center can be doled out to every VM. At VM level, the VM doles out a fixed measure of available managing capability to the individual application administrations (assignment devices). One of the cloud advantages is the likelihood to step by step adjust (scale-up or downsize) the degree of (provisioned) belongings to applications on the way to visit the sorts well-known, which are unsurprising [3]. Versatile (programmed scaling) programs, for instance, net facilitating, content fabric conveyance, and casual companies, can utilize this cloud potential, that's prone to flexibility.

Inexperienced dispensed computing is created no longer absolutely to carry out talented handling and use of registering belongings, but in addition to restrict strength utilization [4]. This is critical for supportable improvement of disbursed computing [5]. Something else, server farms will purpose a massive increment in power utilization [6]. To perform green disbursed computing, server farm belongings have to be handled; that is, cloud property need to be allotted not completely to fulfill QoS stipulations (indicated by way of manner of clients by way of control degree understandings (SLA)), however further to lower electricity utilization [7].Although current virtualization improvements can guarantee the presentation detachment amongst VMs, which percentage a comparable host, due to the forceful solidification and the changeability of the final burden, some VMs may not get the vital degree of belongings while cited. This activates diminishing execution as a protracted way as extended response instances, breaks, or disappointments inside the most pessimistic state of affairs. On this manner, cloud carriers need to control the power-execution tradeoff (i.E., restricting power usage while assembly the QoS situations). On this artwork, the price and energy utilization of time-shared approach emerge as estimated and afterward the rate emerge as displayed within the CloudSim test tool. In mild of the proposed 
version, tremendous situations have been recreated and the outcomes showed that the energy usage is extensive. As a result, cloud carriers have a tradeoff a few of the response time and time-shared arrangement rate.

One of the huge requirements to accept through the use of distributed computing situations is robust QoS [8]. It's miles characterised as a long way because the administration degree understandings (SLA), which portray such characteristics because the throughput, reaction time, or state of no activity conveyed by way of manner of the sent framework [9]. Response time is a measure of time, were given from the interim most of the solicitation accommodations and the main response era. In sensible frameworks, for example, web administrations and ongoing applications, reaction time is the foremost QoS aspect [10]. Response time is an element of burden stress, which may be predicted concerning appearance fees, (as an example, desires each 2d) or the quantity of simultaneous solicitations. Inside the circumstance in which the response time is a higher priority than the particular QoS elements, the framework have to follow time-shared affiliation. The the relaxation of the paper is sorted out as pursues: first we gift related paintings and our fundamental inspirations for this paintings in phase 2. Phase 3 gives the estimating putting transfer fee. Trial effects are then factor through factor in segment 4, ultimately section five finally ends up the paper.

\section{WRITING EVALUATION}

\section{1 title: steady question answers in Inconsistent Databases}

Creator: Marcelo Arenas, LeopoldoBertossi, Jan Chomicki

On this paper we recollect the difficulty of the sensible portrayal of the idea of reliable answer in a social database that can push aside given respectability imperatives. This idea is caught as far due to the fact the attainable constant variations of the database. A technique for processing predictable solutions is given and its adequacy and fruits (for positive instructions of imperatives and inquiries) verified. The approach is predicated upon on an iterative method whose end for some commands of requirements is established too.

Trustworthiness barriers lure a splendid regulating a part of every database software program. Anyhow, it's miles regularly the scenario that their achievement can't be ensured, considering the presence of conflicting database examples. All matters taken into consideration, it's miles critical to apprehend which query solutions are predictable with the honesty obstacles and which can be most in reality now not. In this paper, we provide a practical portrayal of consistent query answers in social databases that is probably conflicting with the given trustworthiness requirements. Instinctively, a response to an inquiry provided to a database that disregards the trustworthiness necessities may be reliable in an specific sense: It should be equal to the ideal response had been given from any insignificantly constant rendition of the first database. We likewise deliver a technique to figuring such answers and show its homes. Based totally on a question $\mathrm{Q}$, the method figures, utilising an iterative device, some other inquiry $\mathrm{T} \omega(\mathrm{Q})$ whose evaluation in a self-assertive, consistent or conflicting, database restores the association of dependable responses to the first inquiry Q. We don't forget using our results in various regions:

Records warehousing: An information distribution middle incorporates facts originating from a substantial range of assets. A number of it typically would no longer fulfill the given uprightness requirements. The standard method is in the long run to smooth the records via manner of expelling irregularities in advance than the facts is placed away in the stockroom . Our results make it plausible to determine out which information is as of now pleasant and preserve to safely evacuate unclean statistics. Similarly, an trade situation receives ability, wherein the irregularities are not evacuated however alternatively inquiry answers are set aside as "predictable" or "conflicting". Along those traces, information misfortune because of information cleaning might be predicted.

Database mix: regularly a huge variety of databases are integrated together to provide a solitary delivered collectively view to the customers. Database incorporation is troublesome because it requires the desires of numerous varieties of inconsistencies of the coordinated databases. One potential inconsistency is due to diverse arrangements of honesty necessities. Except, irrespective of whether or not every coordinated database regionally fulfills a similar uprightness hassle, the requirement might be comprehensively abused. As an instance, severa databases may additionally dole out numerous gives to a similar understudy. Such clashes can also overlook to be settled in any respect and conflicting information can't be "wiped easy" as a consequence of the self-sufficiency of diverse databases. Hence, it's far important to have the choice to discover, given some of neighborhood trustworthiness obstacles, which question answers got here again from the coordinated database are predictable with the necessities and which might be maximum in fact not.

Dynamic and responsive databases: An infringement of uprightness boundaries is probably excellent sufficient beneath the affiliation that it will likely be fixed quicker as opposed to later. For instance, the stock level in a stockroom is probably authorized to fall underneath the crucial least if the critical renewals have been requested. In the course of this quick irregularity, anyhow, inquiry solutions have to provide a sign whether they will be predictable with the imperatives or not. This issue is in particular severe in dynamic databases that allow such consistency slips. The end result of assessing a motive situation this is dependable with the respectability limitations have to be dealt with uniquely in evaluation to the one that is not. The accompanying model introduces the essential instincts behind the concept of predictable query solution. 


\section{PROBLEMS AND CONTRIBUTIONS}

Our persuading packages share the accompanying prerequisites and problems for all intents and reason. In the number one region, it's far insufficient to create a solitary restoration, no matter whether or not it's far about perfect in some properly-characterised enjoy. 2nd, because of the exponential vicinity of viable FD fixes, we can most probably be not able to, or may not have any desire to, produce all fixes. Rather, the check lies in finding a awesome subset of fixes that might created in an powerful way. $1 / 3$, we need to allow purchaser characterized requirements that discern out which sections, tuples, or cells ought to possibly be altered during a repair. In this paper, we suggest a unique technique for records cleansing that suits our inspiring programs and addresses the formerly cited problems. Our method depends on proficiently producing an irregular instance of a considerable repair area. Our precise commitments are as consistent with the subsequent.

We gift a unique area of capacity fixes, called cardinalityset-insignificant, that consolidates the upsides of present spaces: set-negligible and cardinality-minimum. We provide a green calculation for creating an atypical example of cardinality-set-negligible fixes. A tremendous take a look at proper right here is the interchange among infringement of FDs, i.E., solving a tuple that abuses one FD also can gift a few different infringement of each other FD.The number one knowledge in the back of our calculation is to play out the restore each smartphone in flip, alternatively of every tuple in flip. Any other huge check is effectiveness, in moderate of which we gift a device that segments the information case into obstructs that can be steady freely. We depict a sincere alteration of our approach that lets in clients to signify difficult obliges at the association of cells that might in all likelihood be changed all through a repair.We likewise direct an exploratory research to show the versatility of our repair analyzing method.

\section{1 trouble assertion}

Given pretty some practical situations $\Sigma$, which can be conflicting regarding a database occasion I, find masses of minimum try facts fixes and a healing of $\Sigma$ so as to make an $I^{\prime}$ and succinct $\Sigma$ ' that are predictable.

\section{Three.1.1 Contributions}

- a new charge model for information and issue fixes over a database that is conflicting concerning some of requirements.

- A records repair calculation that appears for facts changes with the stop purpose that the imperatives preserve and the restore fee is insignificant. For an utilitarian reliance $\mathrm{F}: \mathrm{X} \rightarrow \mathrm{Y}$ and tuples $\mathrm{t} 1, \mathrm{t} 2$ that abuse $\mathrm{F}$, we are capable of restore those tuples by way of each changing their $Y$ esteems to be the same, or by adjusting their $\mathrm{X}$ esteems to seem as some thing else. In the instances, we necessitate that the information bolster the change. In particular, while converting $\mathrm{X}$ esteems, we simply consider values for $\mathrm{X}$ which is probably upheld by way of awesome tuples.

- A trouble repair calculation that figures out which credit score to add to an vital to decide the irregularity. We pick out out traits for the repair using an adjusted idea of the difference of information between the brand new property and the conflicting FD that measures how properly a trait fixes the irregularity without bringing down the extra in the imperative.

\section{Three.2 Motivating Examples}

Freely of methods we determine to fix infringement, repair structures have showed up in beyond paintings. One is to create a solitary, about ideal repair, as a long way as the amount of erasures or great adjustments (e.G., [6, 12]). For example, we may additionally lean towards restore 2 in Figure 1 in moderate of the fact that it makes a whole lot less adjustments. Some other technique-dependable inquiry replying-methods solutions to chosen commands of questions which may be legitimate in each potential "practical" restore. In Figure1, a steady answer of the inquiry that pick out all tuples with ZIP code 90101, as for the two represented fixes, is $t 4$.

We contend that contemporary methodologies do now not address the requirements of in any occasion the accompanying applications.

\section{Three Notations and Definitions}

Give $\mathrm{R}$ a risk to be a connection on which quite some FDs is characterized. Houses of $\mathrm{R}$ are intended by using manner of $\operatorname{Attrs}(\mathrm{R})=\mathrm{A} 1, \ldots, \mathrm{Am}$. $\operatorname{Dom}(\mathrm{A})$ shows the area of a first-class $A \in A t t r s(R)$. An example I of $R$ is lots of tuples, each taken into consideration considered one of which has an area with the vicinity $\operatorname{Dom}(\mathrm{A} 1) \times \cdots \times \operatorname{Dom}(\mathrm{Am})$. We represent with the aid of manner of DomI (A) the affiliation of estimations of a trait $A \in A \operatorname{trrs}(R)$ that show up in tuples of I (i.E., DomI (A) = ПA(I)). We receive that each tuple in I is associated with an identifier $t$ that closing elements unaltered no matter whether or not or not a part of its trait esteems alternate. We imply thru T IDs(I) the affiliation of identifiers of tuples in I. We allude to a awesome A $\in \operatorname{Attrs}(\mathrm{R})$ of a tuple $\mathrm{t} \in \mathrm{T} \operatorname{IDs}(\mathrm{I})$ as a cell, intended $\mathrm{t}[\mathrm{A}]$.

Each mobile $\mathrm{t}[\mathrm{A}]$ is identified by using the usage of its tuple $t$ and its belongings $A$. The arrangement of all cellular identifiers in I is indicated CIDs(I). We suggest via $\mathrm{I}(\mathrm{t}[\mathrm{A}])$ the estimation of a cell $t[A] \in C I D s(I)$ in an example I.

For two satisfactory devices $\mathrm{X}, \mathrm{Y} \subseteq \mathrm{Attrs}(\mathrm{R})$, a FD $\mathrm{X} \rightarrow$ $\mathrm{Y}$ hangs on an incidence $=\mathrm{X} \rightarrow \mathrm{Y}$, if for every two tuples $\mathrm{t} 1, \mathrm{t} 2$ in $\mathrm{I}$ to such an volume that $\mathrm{t} 1[\mathrm{X}]=\mathrm{t} 2[\mathrm{X}], \mathrm{t} 1[\mathrm{Y}]=$ $\mathrm{t} 2[\mathrm{Y}]$. The association of FDs characterised over $\mathrm{R}$ is meant as $\Sigma$. We receive that $\Sigma$ is insignificant and in cutting-edge shape [2]; each FD is inside the form $X \rightarrow A$, wherein $X$ $\subset \operatorname{Attrs}(\mathrm{R})$ and $A \in \operatorname{Attrs}(\mathrm{R})$. I is conflicting concerning $\Sigma$ inside the event that I damages in any occasion one FD in $\Sigma$.

By means of and huge, a recuperation of a conflicting prevalence I is some different example I 0 that fulfills $\Sigma$. As clarified in segment 1.1, we're capable of actually undergo in mind fixes acquired through a lot of adjustments to I.

\section{Three.Four Measuring Context switch price}

On this region, the proposed technique is supplied. The method is predicated upon at the information expected at various perceptions on the real framework. Within the number one subsection, the real framework model is 
portrayed and in the second subsection the version that is implemented for assignment is delineated.

\subsubsection{Measuring Context transfer fee In real system}

As in step with Ousterhout's approach approaches over and once more deliver a solitary byte message to every other thru channels. In each whole circle correspondence, two placing switches clearly as one observe and one compose framework deliver in each manner will appear. On this studies the time fee of one, 000 complete circle interchanges () became expected, and the time charge of one,000 mimicked complete circle correspondences (), which includes no putting transfer cost, changed into predicted additionally. The at once time charge consistent with putting transfer have become decided as .

A double middle processor modified into used in the research to preserve a strategic distance from the functionality impedance from OS interfere with searching after or from top notch approaches. The providing bureaucracy inside the take a look at have been appointed to the only center. The continuing making plans association turn out to be moreover set by way of setting SCHED_FIFO and giving them the maximum excessive priority. The device in this trial had a CPU with double $2.0 \mathrm{GHz}$ Intel Pentium centers with $2 \mathrm{MB}$ keep. The regular direct setting transfer fee in our framework became three.Nine microseconds.To diploma the impact of information get right of entry to, after every approach were run, it were given to a spread of long entire numbers (lengthy longint) before composing a message to the following machine and later on have become obstructed on the following observe hobby. The all out records were given to via way of every procedure (cluster size) changed at some stage in the various trials.

The examination effects fall into three classifications. In the important class, the cost is normally regular, with placing switch instances extending from $4 \mathrm{~s}$ to eight. $2 \mathrm{~s}$. That is in view that the complete datasets of the two conveying strategies can in form into the reserve, and the setting switch failed to purpose any big store interference.Inside the following type, the dataset of one manner fits inside the reserve, but the joined datasets of the 2 providing bureaucracy don't; the expense of the placing transfer increments notably, from $35 \mathrm{~s}$ to a hundred $90 \mathrm{~s}$, with the enlargement of the showcase length.

\section{SMOOTH QUESTION ANSWERING}

The more part of the writing on records cleansing has centered on growing a spotless case beginning from a grimy one. Anyhow, the problem of describing and recuperating the data inside the first example that can be regarded as perfect has been disregarded. On this segment we've got a observe this trouble, focusing on inquiry replying. All of the more effectively, given an event $\mathrm{D}$, a set $\Sigma$ of MDs, and an inquiry $\mathrm{Q}$ supplied to $\mathrm{D}$, we need to explain the proper responses which may be consistent with $\Sigma$, i.E., that might be returned via an incidence in which every one of the MDs have been carried out. Glaringly, we want to bear in thoughts that there is probably a few such times. This case is just like the one experienced in reliable question replying (CQA) [3, 9, 13], where inquiry noting is described and finished on database cases that can neglect to meet certain terrific uprightness imperatives (ICs). For one of these database case, a repair is an occurrence that fulfills the uprightness barriers and negligibly contrasts from the primary event.

For a given inquiry, a regular solution (a form of positive answer) is characterised because the association of tuples which might be to be had within the convergence of solutions to the question at the same time as supplied to each repair. A much less mainstream non-compulsory is the idea of practicable solution, that is characterised because the association of all tuples that are available inside the reaction to the inquiry while supplied to every repair. A similar semantics for smooth inquiry replying beneath coordinating situations can be characterised. Anyhow, the unfinished request courting $\sqsubseteq$ between a grimy event and its spotless examples devices up a huge distinction between easy occurrences w.R.T. Coordinating conditions and fixes w.R.T. Conventional ICs.Intuitively, a spotless occasion has progressed the records that formerly existed inside the grimy occurrence and made it increasingly more educational and predictable. We'd likely need to painstakingly make the most this halfway request dating and use it inside the meaning of positive and capability solutions. We try this by way of taking the superb decrease positive (glb) and least top sure (lub) of solutions of the inquiry over numerous spotless instances, in vicinity of taking the set-theoretic convergence and affiliation.

Let $\Sigma$ be plenty of MDs, D0 be a database instance, and Q be an inquiry furnished to event D0. We signify sure and capacity answers as follows.CertQ(D0) $=\mathrm{glb} \sqsubseteq \mathrm{D}$ is a $(\mathrm{D} 0$, $\Sigma)$ - easy example. (2)

PossQ(D0) = lub巨Q(D) smooth example. (three)

The accompanying version spurs the ones alternatives. It furthermore suggests that, under no circumstances like a few instances of conflicting databases and predictable inquiry replying, advantageous answers may be very enlightening and giant for databases with coordinating conditions.

Model. Do not forget connection R(name, telephone, cope with), and set $\Sigma$ comprising of the accompanying MDs:

$\phi 1: \mathrm{R}$ [name, telephone, address ] $\approx \mathrm{R}$ [name, telephone, address] $\rightarrow \mathrm{R}$ [address] R[address],

$\phi 2: \mathrm{R}$ [phone, address] $\approx \mathrm{R}$ [phone, address] $\rightarrow \mathrm{R}$ [phone] $\leftrightharpoons \mathrm{R}$ [phone].

Count on that within the filthy incidence D0, confirmed as follows, the accompanying similitudes hold:

"John Doe" ₹ "J. Doe",

"Jane Doe" $\approx$ "J. Doe",

"(613)123 4567" ₹ "123 4567",

"(604)123 4567" ?".

\begin{tabular}{l|l|l|l}
$D_{0}$ & name & phone & address \\
\hline & John Doe & $(613) 1234567$ & Main St., Ottawa \\
& J. Doe & 1234567 & 25 Main St. \\
& Jane Doe & $(604) 1234567$ & 25 Main St., Vancouver
\end{tabular}

Observe that from D0 we can obtain two different (D0, $\Sigma$ )- clean instances $\mathrm{D}, \mathrm{D}^{\prime}$, depending on the order of enforcing MDs.

Published By 


\begin{tabular}{l|l|l|l}
$D$ & name & phone & address \\
\hline & John Doe & $(613) 1234567$ & 25 Main St., Ottawa \\
& J. Doe & $(613) 1234567$ & 25 Main St., Ottawa \\
& Jane Doe & $(604) 1234567$ & 25 Main St., Vanoouver
\end{tabular}

\begin{tabular}{l|l|l|l}
$D^{\prime}$ & name & phone & address \\
\hline & John Doe & $(613) 1234567$ & Main St., Ottawa \\
& J. Doe & $(604) 1234567$ & 25 Main St., Vancouver \\
& Jane Doe & $(604) 1234567$ & 25 Main St., Vancouver
\end{tabular}

Now consider the query Q : $\pi$ address( $\sigma$ name="J. Doe"R), asking for the residential address of $\mathrm{J}$. Doe. We are interested in a certain answer. It can be obtained by taking the greatest lower bound of the two answer sets:

$$
\begin{aligned}
& \mathcal{Q}(D)=\{(\text { (25 Main St., Ottawa") }\}, \\
& \mathcal{Q}\left(D^{\prime}\right)=\{(" 25 \text { Main St., Vancouver" })\} .
\end{aligned}
$$

In this situation, and in step with [6], and the usage of Lemma 1,

We can see that, no matter how we smooth D0, we are able to say with fact that J. Doe is at 25 Main St. Note that the intersection of the two units of solutions settheoretic empty. If we are inquisitive about all possible answers, we are able to take as a minimum an upper limit of two units of solutions, with a purpose to be the union of the 2 in this case.

We outline internet solutions into higher and decrease limits of the solution to the call for for all viable interpretations of the internet from the database example is dirty. This definition is inspired through the equal form of estimates used in the context of a database of facts that is not consistent partial and incomplete and information alternate. These higher and lower limits can provide beneficial statistics about the value of aggregate capabilities, such as the variety and matter.Definition 6. To query $Q$ pose for a pattern database and a fixed of MDs D0 $\Sigma$, the answers are dictated by two limits as

$$
\operatorname{Clean}_{\mathcal{Q}}\left(D_{0}\right)=\left(\operatorname{Cert}_{\mathcal{Q}}\left(D_{0}\right), \operatorname{Poss}_{\mathcal{Q}}\left(D_{0}\right)\right) .
$$

\subsection{Various Types of Repairs.}

In this segment, we present the cardinality of the setimprovement is negligible, which is targeting to find some kind of harmony between the "least change" cardinality metrics-not important and "important changes" setminimalityDEFINITION standard-Set-Cardinality 6. Minimal Repair Repair I 0 I was kardinalitas- set-ignored me "no improvement I 00 I with the ultimate goal that $\Delta$ (I, I ') $\subset \Delta(\mathrm{I}, \mathrm{I}$ ').

That is, the fix I $0 \mathrm{I}$ is the cardinality of the setinsignificant iff there is a part $\mathrm{C}$ of the cells changed in I 0 can be restored to their unique qualities in I without abusing $\Sigma$, regardless of whether the cells in $\Delta$ (I, I0) $\backslash \mathrm{C}$ which is converted to a different quality. In Figure 2, we show various types of repair of my case, the changed cells turns gray. Repair I1 and I 2 is the cardinality-is not significant given the fact that there are other improvements have cells that are less unchanged. Obviously, I1 and I2 is an additional cardinality - set insignificant and setminimality.
I3 set-not significant given the fact that back in one of the cells turn into quality I would misappropriate $\mathrm{A} \rightarrow \mathrm{B}$. Then again, I3-set-cardinality not insignificant (or cardinalityignored) in light of the fact that the change $\mathrm{t} 1$ [B] for 3 and back $\mathrm{t} 2$ [B] for 3 provides a fix of I. I4

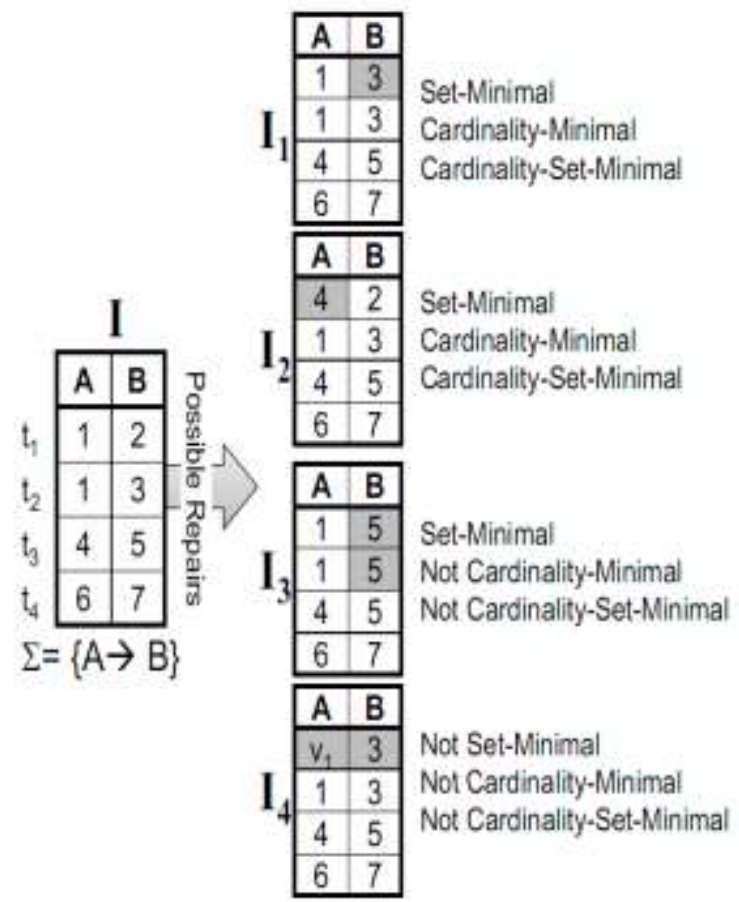

The relationship among the various definitions of minimal repairs is depicted in Figure 3 and described in the following lemma (whose proof is in Appendix C.1).

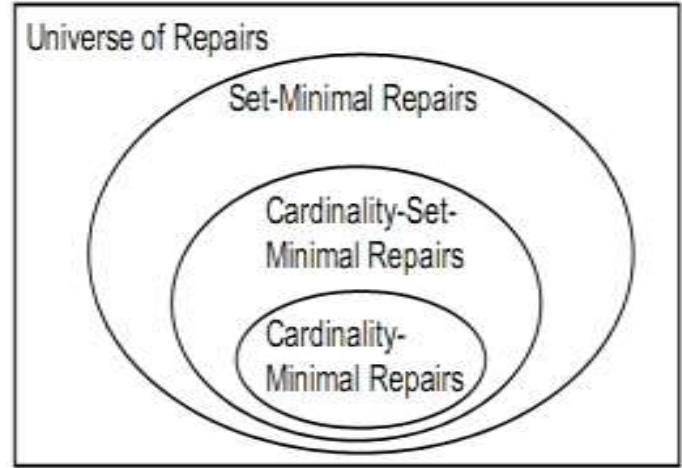

LEMMA 1. The set of cardinality-minimal repairs is a subset of cardinality-set-minimal repairs. Moreover, the set of cardinality set-minimal repairs is a subset of set-minimal repairs.

\section{ALGORITHM FOR GENERATING REPAIRS \& RESULTS}

Note that for any tuples $\mathrm{t} 1, \mathrm{t} 2$ in violation of the FD $\mathrm{X} \rightarrow$ $\mathrm{A}$, ie, that agree on $\mathrm{X}$, however not $\mathrm{A}$, a change this is enough to generate improvements: we both can alter t1 [A] in order that together with $\mathrm{t} 2[\mathrm{~A}]$ ( or in any other case), or adjust the attributes $B \in X$ both $t 1$ or $t 2$ so that $t 1[B] 6=t 2$ [B]. 
Generalizing this commentary, if one set of cells $\mathrm{C}$ does not violate any $\mathrm{FD}$, consistency set $\mathrm{C} \cup \mathrm{C}$, for each mobile $\mathrm{C}$, it is able to usually be enforced by using modifying $\mathrm{C}$ (if necessary). Our algorithm is primarily based on this commentary; preserve a smooth set of cells which can be extended in the course of each iteration by using incorporating a randomly decided on cellular is new and makes the necessary changes to the cellular is inserted. We first outline a smooth set of cells in my instance as follows. A set of cells $\mathrm{C}$ in the instance I smooth if there's as a minimum one improvement $I$ ' $\in$ Repair (I) (any kind) so $\forall \mathrm{C} \in \mathrm{C}, \mathrm{I}(\mathrm{C})=\mathrm{I}(\mathrm{C})$.

That is, one set of cells in my example is smooth if the values of the cellular I could continue to be unchanged while getting repairs I. Note that it is not sufficient to verify that the cells in $\mathrm{C}$ on my own did no longer violate any FD. As an instance, recollect Figure 4, which shows a subset of cells in a sample. Assume that we need to determine whether or not the cells are shown internet. Although the cells do no longer violate the FD proven in $\Sigma$, no development may incorporate the current values of the cells regardless of the values of other cells. This is because $t$ [A] $=\mathrm{t} 2[\mathrm{~A}]$ implies $\mathrm{t} 1[\mathrm{C}]=\mathrm{t} 2[\mathrm{C}]($ by $\mathrm{A} \rightarrow \mathrm{C})$ and $\mathrm{t} 2[\mathrm{~B}]=\mathrm{t} 3$ $[B]$ means that $\mathrm{t} 2[\mathrm{C}]=\mathrm{t} 3[\mathrm{C}]$ ( by means of $\mathrm{B} \rightarrow \mathrm{C}$ ), but $\mathrm{t} 1$ $[\mathrm{C}]=\mathrm{t} 3[\mathrm{C}]$.For systematically determine whether or not a fixed of clean cells, we need to keep music of equivalence training. We declare equivalence relation $\mathrm{E}$ (ie, a fixed of equivalence lessons). We specific ec (E, Ci) equivalence class $\mathrm{E} \in \mathrm{E}$ which cells belong $\mathrm{Ci}$. We imply by way of merging two equivalence training in $E$ replace it with a new equivalence elegance is the same with their union. Algorithm 1 describes a way to establish equivalence relation $\mathrm{E}$ is a set of cells $\mathrm{C}$ in my instance.

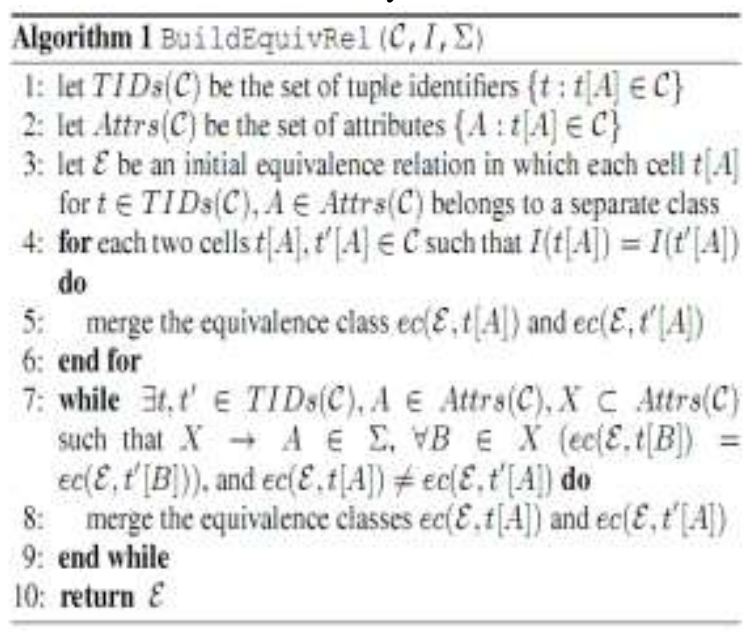

Having run Algorithm 1 to generate $\mathrm{E}$, a set of cells $\mathrm{C}$ is clean in I, denoted Is Clean (C, I, E), if every two cells in C that belong to the same equivalence class in $\mathrm{E}$ have the same value in I. Formally (see Appendix C.2 for a proof):

THeores 1. IsClean $(\mathcal{C}, I, \mathcal{E})$ is True iff $\nexists C_{i}, C_{j} \in \mathcal{C}$ such that $e c\left(\mathcal{E}, C_{i}\right)=e c\left(\mathcal{E}, C_{j}\right) \wedge I\left(C_{i}\right) \neq I\left(C_{j}\right)$

Figure four indicates the case of unclean cells. Cells that have a place with a similar equivalence magnificence seem within the identical square form. At first, every series of cells that have similar structures worth equivalence instructions. At the time, we take advantage of FD in $\Sigma$ identicalness magnificence to any other interpret. For instance, cells $\mathrm{t} 1$ [A] and $\mathrm{t} 2$ [A] are in the identical equivalence elegance, and therefore $\mathrm{t} 1[\mathrm{C}]$ and $\mathrm{t} 2[\mathrm{C}]$ is set inside the identical comparison class. In the conclusive results, $\mathrm{t} 1[\mathrm{C}]$ and $\mathrm{t} 3[\mathrm{C}]$ have a place with the identical magnificence but the comparability of their superb nice, indicating that the cells analyzed are unclean.

We are making ready to reveal the calculations to provide improvements arbitrary set of rules 2 . Cells are embedded into CleanCells set in arbitrary order (line 4 ). In every cycle, the exam of whether or not CleanCells perfect calculations (strains five,6). In the case of CleanCells unclean, cells which have been embedded into CleanCells, show $\mathrm{t}$ [A], is amended as chasing. Let Ep be identicalness connections to cells CleanCells earlier than embeddings t [A] (line 7). We changed $\mathrm{t}[\mathrm{A}]$ for the worth that meet equality connections Ep.

We appear in Appendix B that the multifaceted nature of the asymptotic of Algorithm 2 is $O(m 2-$ three $n \log n)$, where $n$ is the range of tuples, and $m$ is the range of homes. Likewise, the speculation that accompanies the display correctness of Algorithm 2 (affirmation in Appendix C.3). Hypothesis 2. Every occasion that may be created by way of Algorithm 2 is to enhance the set-insignificant of my case information w.R.T. $\Sigma$. All upkeep are not noted cardinality of the set information events I w.R.T. $\Sigma$ may be made by means of Algorithm 2.

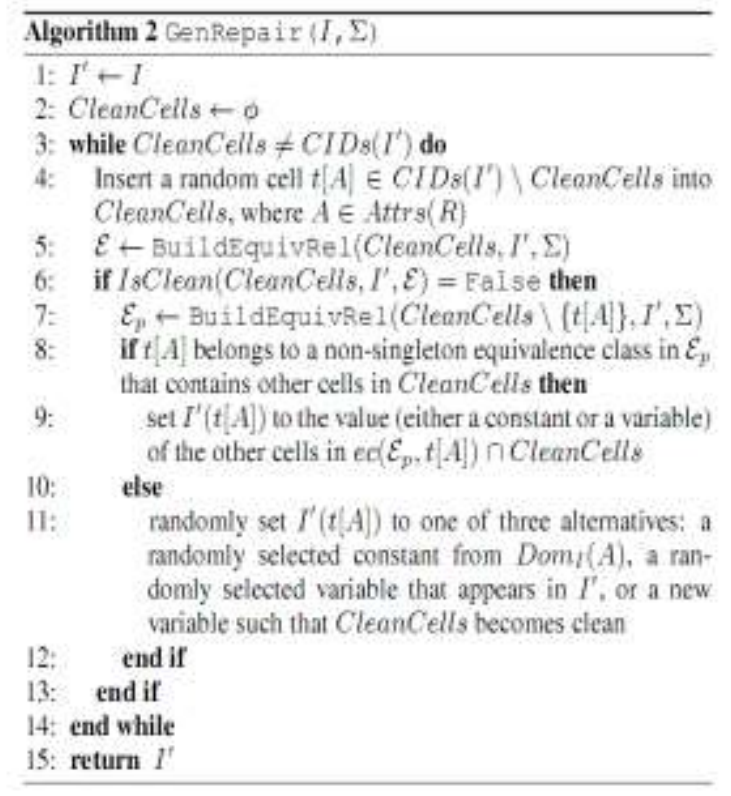

\subsection{Block-wise Repair Generation}

In this stage, we improve the presentation of yield support through my model approaching apportioning into squares autonomously redressed. We are currently not

We saw that the alter line eleven Algorithm 2 to handiest set a fresh out of the plastic new factor to the T cell [A] (ie, the third turn), ensure that the $\mathrm{T}$ cell $[\mathrm{A}]$ can in no way, shape or form indistinguishable from different telephones in the A. subsequently, $t$ [A] can in no way, shape or form be a piece of an offense for any FD wherein An is in wording

Published By: Blue Eyes Intelligence Engineering 
leftand. We look for the counsel of the changed model of Algorithm 2 (ie, where the line 11 controlled with an unobtrusive 0.33 other options) as Algorithm ModGenRepair. The comparability connection $\mathrm{E}$ is made of the estimation of the phone in the main (ie, BuildEquivRel (CID (I), I, $\Sigma$ )) bunch of cells into the directions of balance so every phone has a similar incentive during the execution of ModGenRepair handiest on the off chance that they are remembered for the correspondence of tastefulness the equivalent (we give proof in Appendix C.Four).

It pursues that the cells have a place with a one of a kind identicalness class could once don't have a similar expense. For instance, in Figure 6, cells t1 [C], t2 [C] and t3 [C] identicalness class has a place indistinguishable, which implies that they may have similar qualities in a portion of the created upkeep. On the contrary side, $\mathrm{t} 1$ [B] and $\mathrm{t} 2$ [B] is a stand-out preparing of correspondence, which implies that they can in no way, shape or form be the equivalent values.We utilizing identicalness connection $\mathrm{E}$ to segment the information test with the goal that two tuples which have a place with one of the square sort can never have a similar incentive for the left half of qualities $X$, for all $X \rightarrow A \in \sigma$. Accordingly, any infringement of the FD during the fix I can not traverse various squares. In various expressions, fixing each square in a steady progression outcomes in fix I.In input test Algorithm 3, we disclose how to parcel my model be a rigid out of the squares traces that can be fixed independently utilizing ModGenRepair calculation with the goal that one can reestablish the example I.In Pictures, we show a case of dividing a model. At first, the equality connection is based on the information test by applying BuildEquivRel (CID (I), I, $\Sigma$ ). Every polish uniformity spoke to as a square shape which encompasses the exquisite patrons. We start by setting any supplier to a different segment (eg, cell t1 [A] have a place with P1, and versatile t2 [A] have a place P2). For each

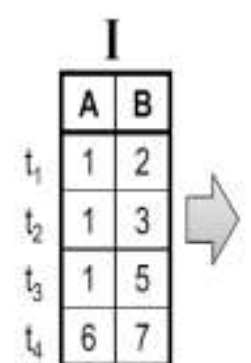

$\Sigma=\{A \rightarrow B\}$

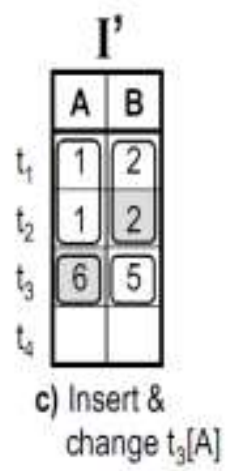

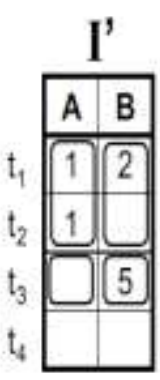

a) Insert $t_{1}[A]$, $t_{1},[B], t_{2}[A], t_{3}[B]$

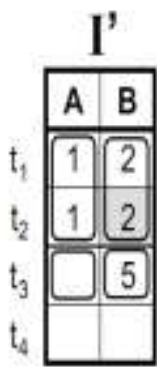

b) Insert \& change $t_{2}[B]$

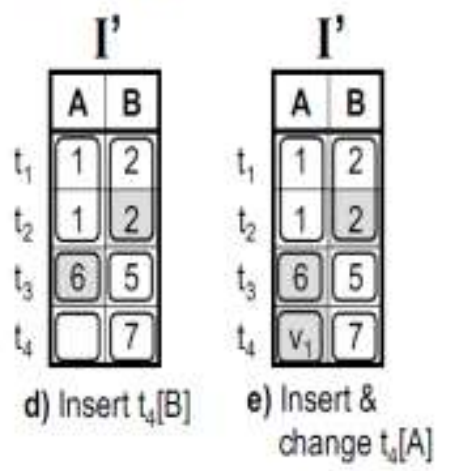

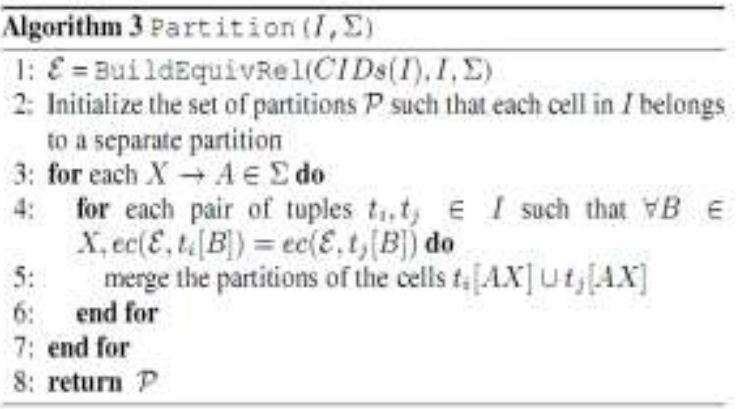

We display in Theorem three that the field is made via Algorithm 3 may be constant independently utilize ModGenRepair.THEOREM Algorithm 3. The rectangular of an incident I developed through partitioning algorithm can be constant independently make use of ModGenRepair set of rules if you want to improve my chance

The information at the back of the boom within the exhibition are as in line with under. We modified the difficulty of fixing a case opposite to the restore container infinite Littler. Because the largest a part of an occasion smooth records in lots of situations, many cells that aren't associated with the infringement, and consequently they'll not be accumulated with

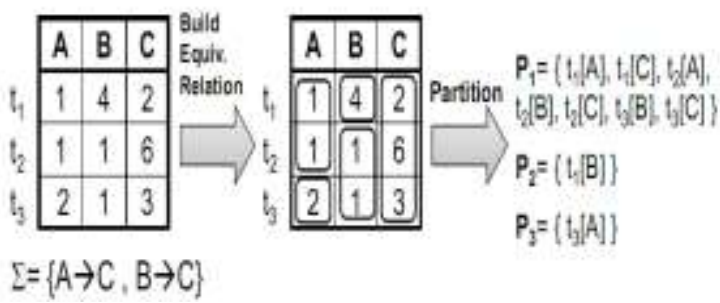

Other cells throughout the partition step (ie, they belong to a unmarried block). Unmarried block is easy and does no longer ought to be processed via algorithms ModGenRepair. This appreciably reduces the overhead put clean cells into cells and comparing whether cell easy clean is smooth.

\section{TAKE A LOOK AT OF EXPERIMENTAL}

In this segment, we can communicate analytical models described in the preceding section. As a centering tool is a cloud computing environment, it is a lot vital to assess on a big-scale infrastructure. Therefore, the important records with 500 host heterogeneous body turns into a simulation. Each host to be a version to have a dual-core CPU; the general overall performance of every middle thereof is equal to one thousand million orders in accordance with the second one (MIPS), a four GB RAM, 2 MB of cache reminiscence, and $1 \mathrm{~TB}$ storage. Energy intake with the assist of the host defined consistent with the model set out within the previous segment. Primarily based on this version, the quantity of energy intake of $210 \mathrm{~W}$ at $0 \% \mathrm{CPU}$ usage of as much as $300 \mathrm{~W}$ with $100 \%$ CPU usage. Each VM calls for one center with a 250 MIPS CPU, $128 \mathrm{MB}$ RAM, and 1 GB storage. The customers submit a request for 


\section{A COST-BASED MODEL FOR DATA REPAIRING/IN CLOUD DATABASE}

the provision of one thousand heterogeneous VMS-8000. For the version of CPU usage, each VM strolling a software that makes use of the net given a uniform random variable workload and make contact with for a 10,000- hundred, 000 MIPS. Securities based totally on the implied fee of going for walks each check five times.

Table 2: Host Parameters.

\begin{tabular}{lcc}
\hline & Host parameter in data center & Value \\
\hline 1 & Number of hosts & 500 \\
2 & Type of CPU & Dual core \\
3 & Each core performance & $1000 \mathrm{MIPS}$ \\
4 & RAM & $4 \mathrm{~GB}$ \\
5 & Cache memory & $2 \mathrm{MB}$ \\
6 & Storage & $1 \mathrm{~TB}$ \\
7 & Power consumption by each host & $210-300 \mathrm{~W}$ \\
\hline
\end{tabular}

Table 3: VM Parameters.

\begin{tabular}{lcc}
\hline & VM parameter & Value \\
\hline 1 & CPU & one CPU core with 250 MIPS \\
2 & RAM & $128 \mathrm{MB}$ \\
3 & Storage & $1 \mathrm{~GB}$ \\
4 & Number of VMs & $1000-8000$ \\
\hline
\end{tabular}

In Figures 3, 4, and 5 the dataset size of applications is zero. Hence, these charts demonstrate the indirect cost.

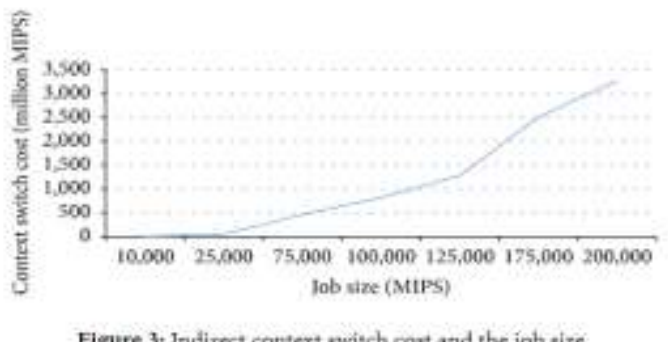

Figure 3t Indirect context witch coxt and the jobs size.

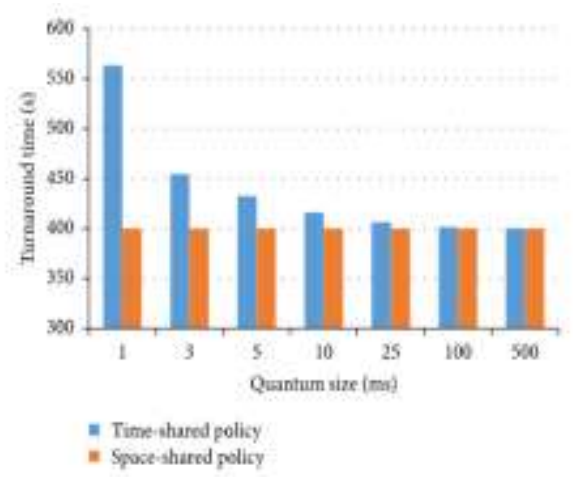

Figure 4t Indirect contest witch cont and the vise of quastum paramest.

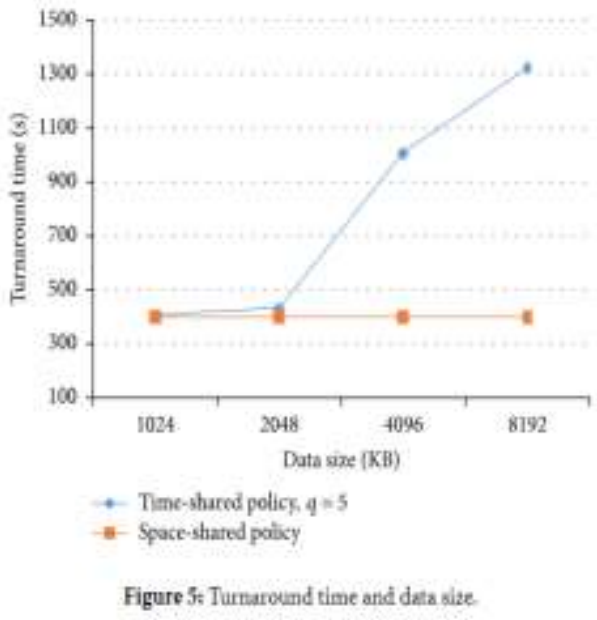

Figure three well-knownshows the rate of time-shared approach as the size of the occupations is expanded. In this discern, pivot speaks to paintings size and hub speaks to the additional price introduced approximately by means of putting switch overhead, given in MIPS.

As can be seen, if the pastime size builds, the price of time-shared approach will likewise increment. This is because of the manner that the larger employments reason greater placing switches than littler occupations. In the cloud condition, the activity length is sufficiently huge. For this situation, the quantum length is $5 \mathrm{msec}$.

Next investigation become intended to assess the impact of the quantum parameter at the turnaround time for timeshared association. Turnaround time is the degree of time between the accommodation of a vocation and its fruits. From the perspective of a selected hobby, the considerable preferred is to what quantity it takes to execute that pastime. In a non-stop or intuitive framework, as an instance, some software in geospatial sciences, usage of turnaround time might not be a respectable measure [33]. Frequently, an profession can supply some outcomes and yield them, and in a while it may keep on growing new consequences even as beyond effects are being yield to the consumer. Thus, any other model is the all out time from the accommodation of a vocation till the number one response is created (reaction time). Figure four suggests that the turnaround time of occupations in the time-shared arrangement increments as the quantum parameter diminishes.

In this analysis, the hobby period is $100,000 \mathrm{MI}$ and the amount of VMs is 4,000. An enlargement within the quantum parameter adds to a lessening inside the timeshared approach fee. Ascertaining the precise degree of quantum parameter is a multicriteria streamlining trouble.Increasing the quantum length will decrease the turnaround time and increment ordinary reaction (time from accommodation until the primary reaction is introduced), although an abatement within the quantum parameter activates a lower within the response time and an ascent in the time-shared strategy value. Consequently there exists a tradeoff between the fee of the placing switch and the reaction time. In the cloud circumstance, both the fee and

Published By: Blue Eyes Intelligence Engineering 
the reaction time are full-size. Hence, it is necessitated that the appropriate traits be chosen for this parameter. In the event that the quantum parameter is sufficiently large, at that point the time-shared approach will become the spaceshared strategy. In this situation, in a length reduce work can gather the entire time fundamental for execution.

\subsection{Effect of Data Access}

In this segment, the statistics get to affect is regarded. As may be visible, the information get to fee of the time-shared arrangement is probably excessive.Figure five shows the information size addition effect inside the turnaround time when the quantum parameter is five msec. The time-shared method bend form is steady with Section 3 results. The timeshared arrangement bend has 3 areas. In the essential locale the bend is commonly stage. This is considering the fact that the entire datasets of processes can fit into the store, and the putting transfer doesn't result in any outstanding reserve impedance. In the second one locale the dataset of 1 procedure fits inside the reserve, yet the consolidated dataset of all techniques does not; the fee of the setting switch increments appreciably with the addition of the cluster length.

Figure 6 suggests the turnaround time for specific quantum parameters as the facts length increments. As may be watched, the quantum parameter is basic for the timeshared approach fee. The price will increment notably, on circumstance that the quantum parameter is little and that the information size is better than that of the store. Henceforth, so one can decrease the time-shared association price, the quantum parameter must be sufficiently huge, prompting a diffusion inside the reaction time. Along those traces, there will be a tradeoff between the rate and the QoS in this circumstance.

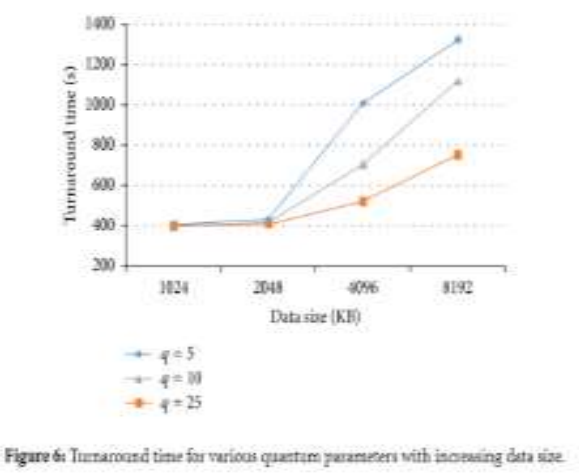

This is because of the fact that a big variety of VMs motive of context switches from a small quantity of VMs. In a cloud environment, the number of VMs is pretty huge. In this test, the running length is a hundred,000 MI and is 5 msec. Thus, if the cloud company decided to consolidate VMs to reduce energy consumption, they need to remember the consolidated cost VMS.

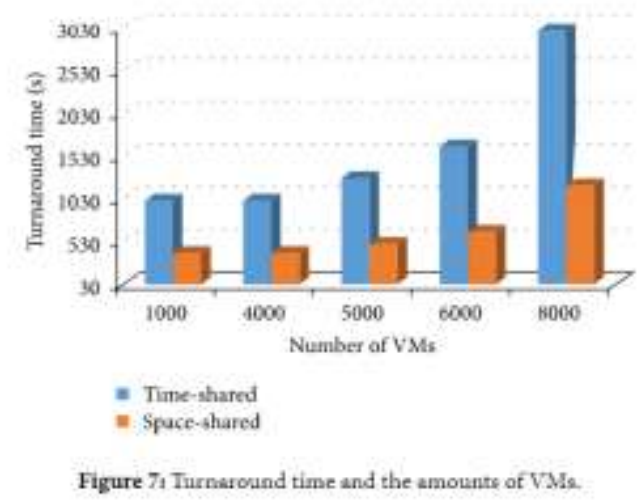

\section{CONCLUSION}

In this paper, we present some other process to restore issue primarily based database, where we create an arbitrary example of ability upgrades. We painting the acknowledgment of this system as regards to Functional Dependencies, given a unique development that consolidates the highlights of two understood present space: insignificant units and cardinality. We additionally stretched out examining calculation to empower customer characterized tough requirements that signify a lot of cells that should stay unaltered throughout the restoration manner. Our exploratory exam shows that the parcel test contribution to obstructs that may be adjusted freely create orders greatness execution gains.

\section{FUTURE ENHANCEMENT}

A massive direction for destiny work is to plot new inspecting calculation improvements for extraordinary types of uprightness limitations and facts first-rate requirements, and comprehend the correct restore space.

\section{REFERENCES}

1. UIS Data Generator http://www.Cs.Utexas.Edu/clients/ml/puzzle/records.Htm

2. S. Abiteboul, R. Body, and V. Vianu.Foundations of Databases.Addison-Wesley, 1995.

3. F. N. Also, P. G. Kolaitis Afrati. Look at enhancements in the database is conflicting: calculations and intricacy. In ICDT, pages 31-41, 2009.

4. M. Fields, L. E. Bertossi, and J. Chomicki. Reliable hobby answers inside the database is conflicting. In PODS, pages 68-seventy nine, 1999.

5. O. Benjelloun, A. D. Sarma, A. Y. Halevy, and J. Widom. ULDBs: Database withuncertainty and plunge. In VLDB, pages 953-964, 2006.

6. P. Bohannon, M. Flaster, W. Fan, and R. Rastogi. A model-based totally and heuristic financially savvy to restore troubles with the adjustment esteem. In SIGMOD, pages 143-154, 2005.

7. J. Chomicki and J. Marcinkowski. Insignificant alternate aid respectability making use of tuple cancellation. Data and Computation, 197 (half): 90-121, 2005.

8. J. Chomicki, J. Marcinkowski and S. Staworko. Processing solution dependable hobby using hypergraphs battle. In CIKM, pages 417-426, 2004 